\title{
Strain rate dependence in plasticized and un-plasticized PVC
}

\author{
M.J. Kendall and C.R. Siviour \\ Department of Engineering Science, University of Oxford, Parks Road, Oxford OX1 3PJ, UK
}

\begin{abstract}
An experimental and analytical investigation has been made into the mechanical behaviour of two poly (vinyl chloride) (PVC) polymers - an un-plasticized PVC and a diisononyl phthalate (DINP)-plasticized PVC. Measurements of the compressive stress-strain behaviour of the PVCs at strain rates ranging from $10^{-3}$ to $10^{3} \mathrm{~s}^{-1}$ and temperatures from -60 to $100^{\circ} \mathrm{C}$ are presented. Dynamic Mechanical Analysis was also performed in order to understand the material transitions observed in compression testing as the strain rate is increased. This investigation develops a better understanding of the interplay between the temperature dependence and rate dependence of polymers, with a focus on locating the temperature and rate-dependent material transitions that occur during high rate testing.
\end{abstract}

\section{Introduction}

The mechanical properties of polymers are studied because of their widespread scientific and industrial importance, as seen in the automotive, aerospace, military, and medical industries. Most polymers exhibit time dependent mechanical behaviour, as evidenced by rate-dependent elastic moduli, yield strength and post-yield behaviour. This rate and temperature sensitivity is seen to change in different temperature and rate regimes (with sensitivity increasing at higher rates of strain) depending on different molecular mobility mechanisms being activated. In several polymers, the sensitivity is understood to increase at higher rates and/or lower temperatures because of a lack of secondary molecular mobility ( $\beta$-motions), which causes increased strength and stiffness [1-8]. Alternatively, the increased strength in more rubbery or elastomeric polymers is accounted for by the change in molecular mobility during the glass-transition (or $\alpha$-transition). These transitions are usually seen within polymers at lower temperatures, but can be shifted in temperature by changes in strain rate, as the transition increases in temperature with increasing strain rate $[1,2,5,6]$.

The capability to alter these molecular-level transitions through the incorporation of additives (i.e. plasticizers) offers new opportunities to understand the mechanical properties of polymers. In many applications in which poly(vinyl chloride) (PVC) is used, it is blended with a plasticizer such as diisononyl phthalate (DINP). The plasticizer is used to enhance ductility and decrease properties such as the yield stress and stiffness. By changing the amount of an additive the temperature- and ratedependence is changed, allowing one to better analyse and compare the mechanisms that govern these dependencies. Thus materials such as PVC have the potential to offer great insight into understanding the interplay between temperature and rate dependence of polymers.

This paper investigates the high strain rate mechanical behaviour of two polymers, un-plasticized PVC (PVC) and a diisononyl phthalate (DINP)-plasticized PVC. Experiments have been performed over a range of strain rates at room temperature and over a range of temperatures at one quasi-static rate. The material transitions in strain rate are compared to those expected from the low rate experiments and Dynamic Mechanical Analysis (DMA) data. This shows how time-temperature equivalence can be utilized in order to predict the strain rate at which certain molecular transitions (glass and $\beta$ ) occur when shifted during high rate deformation, and thus better understand rate dependence in these materials.

\section{Experimental description}

Two kinds of PVC were chosen for investigation in this study: one (un-plasticized) manufactured by Solvin using the resin Solvin DGA166-09 (will be referred to as 'PVC'), and one plasticized PVC (referred to as 'PPVC') Solvin 264-05PC, with a high plasticizer level of 60 parts per hundred resin (phr); DINP was used as the plasticizing agent. The PVC came as pressed plates with dimensions of $11.5 \times 20 \mathrm{~cm}$ and a thickness of $2.5 \mathrm{~mm}$. These plates were machined to the specified specimen geometries, such that the axis of the specimen in the loading direction was perpendicular to the plane of the plate.

In both the high rate and low rate compression tests, the specimens were cylinders of $2.5 \mathrm{~mm}$ length and $5 \mathrm{~mm}$ diameter. These dimensions helped reduce effects of inertia and wave propagation in high rate testing [10]. At the highest rates for PPVC $\left(2930 \mathrm{~s}^{-1}\right)$ and PVC $\left(4360 \mathrm{~s}^{-1}\right)$ with the specimens having initial lengths of $2.5 \mathrm{~mm}$, inertial contributions of $19 \mathrm{kPa}$ and $75 \mathrm{kPa}$ were calculated. These inertial contributions are negligible compared to the room temperature flow stress of $17 \mathrm{MPa}$ for PPVC and $150 \mathrm{MPa}$ for PVC. The specimen sizes were used in the quasistatic testing in order to allow for a more straightforward comparison between rates. All specimens were lubricated on both faces with a thin layer of petroleum jelly [11], and little to no barrelling was observed.

Quasi-static compression tests at room temperature were performed on PVC and PPVC over three decades of strain rate, ranging from $10^{-3}$ to $10^{-1} \mathrm{~s}^{-1}$, using an Instron testing machine. Quasi-static compression experiments conducted in the range of -60 to $100^{\circ} \mathrm{C}$ were performed in the same machine using an environmental chamber. Force measurements were taken from the load 
cell and displacement measurements from a clip gage extensometer attached to the loading platens. Temperature readings from the environmental chamber were ensured by the use of thermocouples attached to the platens adjacent to the specimenplaten interface.

High strain rate testing was conducted on a steel splitHopkinson pressure bar (SHPB) apparatus for PVC and a magnesium SHPB apparatus for PPVC. The striker bar, incident bar, and transmitted bars all had diameters of $12.7 \mathrm{~mm}$, with lengths of $0.48 \mathrm{~m}, 1.0 \mathrm{~m}$, and $0.5 \mathrm{~m}$, respectively. The strain gauges were placed $0.5 \mathrm{~m}$ from the specimen-bar interface on the incident bar and $50 \mathrm{~mm}$ from the specimen-bar interface on the transmitted bar. Dynamic equilibrium was confirmed after each test by calculating force-time profiles for both specimen bar interfaces [12].

DMA testing was conducted in order to understand the rate and temperature dependence of the molecular mobility transitions. These experiments were carried out on a TA Instruments DMA Q800. The rectangular specimens had dimensions of $2.5 \mathrm{~mm}$ height, $10 \mathrm{~mm}$ width, and $35 \mathrm{~mm}$ length, and were used in the dual-cantilever mode of the DMA machine with a set amplitude of $10 \mu \mathrm{m}$. Tests were performed at temperatures ranging from -100 to $100^{\circ} \mathrm{C}$, and frequencies of $0.1,1$, and $10 \mathrm{~Hz}$.

\section{Results}

The stress-strain behaviours of PVC and PPVC under uniaxial compression are presented in figures 1 and 2 . It should be noted that in all the data presented, the end of the stress-strain curve represents the end of the loading, and are not a feature of the specimen response. The results in figure 1 describe the behaviour of both materials at 8 different strain rates at room temperature. PVC exhibits glassy polymer behaviour for all of the tested strain rates. The adiabatic heating effect of the polymer is seen in the large amount of strain softening after yield during the high rate tests. The effect of the glass transition is seen in figure 1(b) for PPVC by the change in its response going from a rubbery response to a more glassy response at higher rates. This is an effect of the glass transition, or $\alpha$-transition shifting to a higher temperature. The behaviour of both materials depends strongly on strain rate, as seen by the change in yield stress, here defined as the peak stress, with increasing strain rate.

The temperature dependence of both materials under quasi-static loading is seen in figure 2. In PVC's response, the effect of the glass transition is seen at approximately $80^{\circ} \mathrm{C}$, which is consistent with the DMA data. At $100^{\circ} \mathrm{C}$ the polymer exhibits a more rubbery response, similar to that of PPVC. The temperature dependence of PPVC illustrates the material transitioning through the glass transition temperature, offering an array of responses throughout the range of temperatures. PPVC's sub-room temperature glass transition is seen at approximately $-40^{\circ} \mathrm{C}$, after transitioning from a more leathery response to a response much like PVC or that of a glassy polymer. It is worth noting the distinctive similarity between PPVC's response at $-20^{\circ} \mathrm{C}$ and strain rates centred at $2500 \mathrm{~s}^{-1}$. These similarities highlight the interplay between temperature and strain rate in a materials response and offers

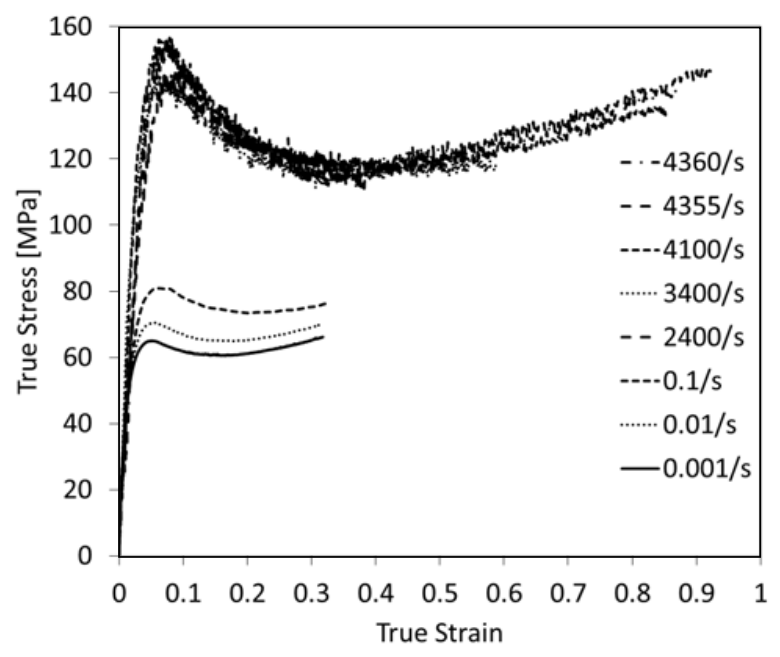

a

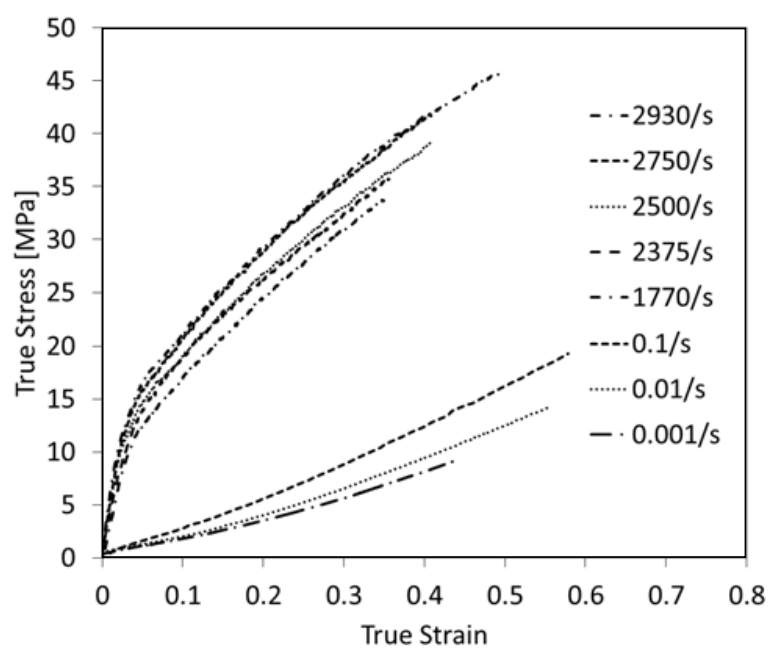

b

Fig. 1. PVC (a) and PPVC (b) stress-strain behavior in uniaxial compression at room temperature over a range of strain rates.

opportunities in analysis, as well as relating well to the time-temperature superposition method [1] which will be discussed in greater detail below.

\section{Discussion}

The dependence of PVC and PPVC's yield strength upon temperature and strain rate is summarized in figures 3 and 5. These curves make the material transitions, which occur at high strain rate/low temperature, clear ( $\alpha$ and $\beta$ in PVC and $\alpha$ in PPVC). A comparison of this data and DMA data is useful in predicting the transitions and the rate at which they occur. PVC's yield stress dependence upon temperature, as seen in figure 3 , is very similar to the shape of the curve produced in DMA when measuring the dependence of the storage modulus with respect to temperature, presented in figure 4 , illustrating the consistency of and confirming the $\alpha$ and $\beta$ transitions in the uniaxial compression data and the DMA data. 


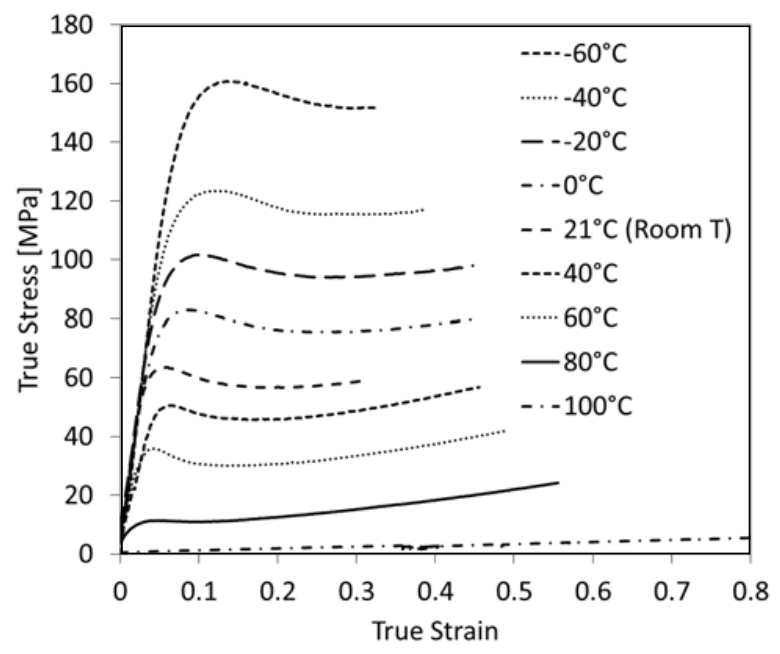

a

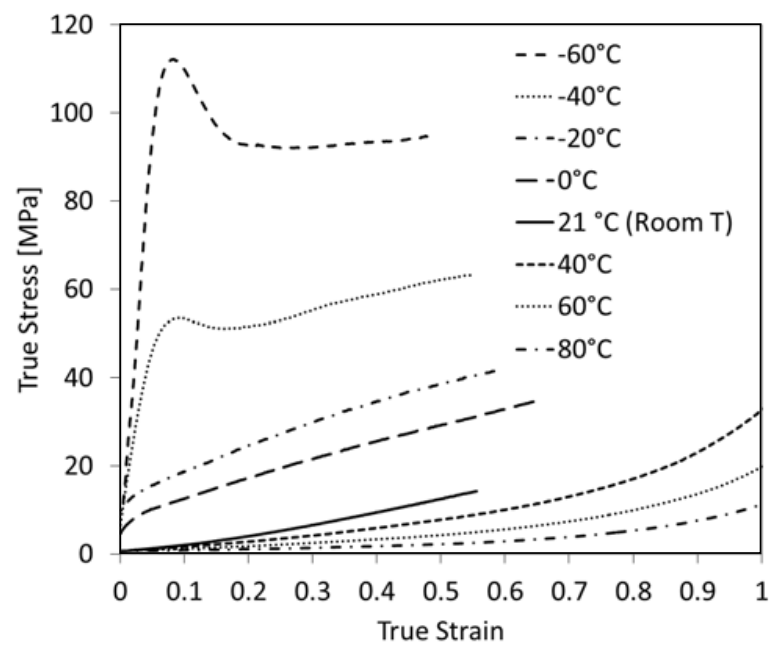

b

Fig. 2. PVC (a) and PPVC (b) stress-strain behavior in uniaxial compression over a range of temperatures. All tests were conducted at a strain rate of $10^{-2} \mathrm{~s}^{-1}$.

From time-temperature equivalence we are able to predict the strain rate at which the $\beta$ transition begins to affect the results from room temperature testing on PVC-seen as the point at which the polymer's strength has an increase in sensitivity to strain rate. By assuming the $\beta$ transition temperature increases linearly with the logarithm of strain rate, a line fitted to the locations of the beta transition temperatures in DMA testing is extrapolated to predict the location of the strain rate (at room temperature) at which the transition occurs. The bets transition temperatures obtained from the DMA data are indicated in figure 4 . The three frequencies are converted to approximate strain rates using

$$
\dot{\varepsilon}=\frac{\frac{d_{0}}{l_{g}}}{\frac{1}{4} \frac{1}{\omega}}
$$

where $d_{o}$ is the displacement amplitude, $l_{g}$ is the specimen gage length and $\omega$ is the test frequency [9]. Figure 6 shows the dependence of the $\beta$ transition temperature on strain

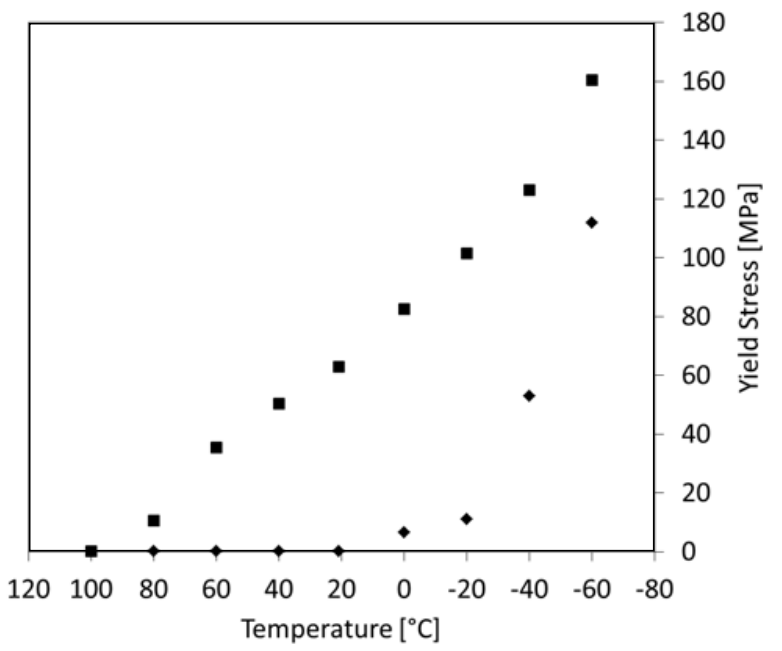

Fig. 3. PVC (squares) and PPVC (diamonds) yield stress dependence on temperature. Note the reverse temperature axis.

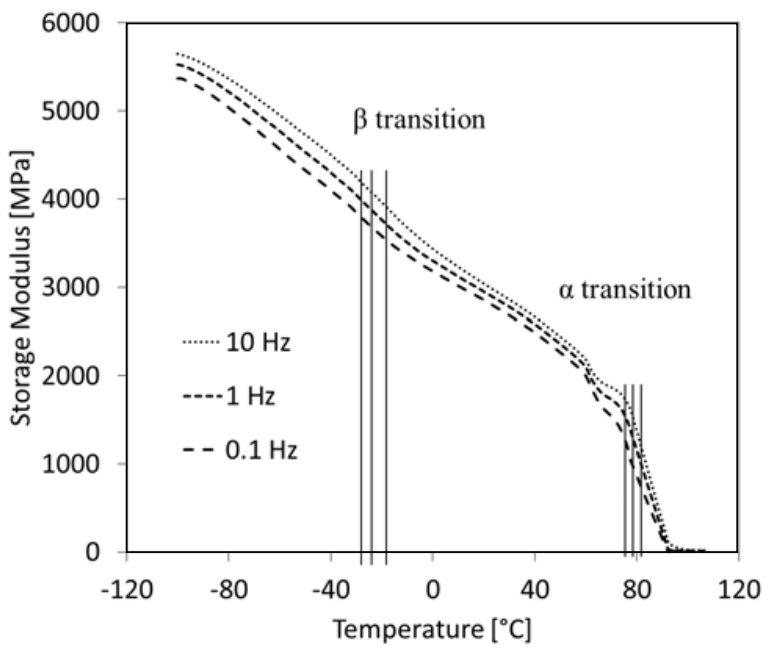

Fig. 4. DMA data for PVC: temperature dependence of the storage modulus at 10,1 , and $0.1 \mathrm{~Hz}$.

rate and extrapolates the dependence seen in the DMA data up to room temperature, or equivalently, high strain rates.

The prediction of the beta transition is analogous to the linear mapping technique in equation (2) [1],

$$
T-T_{0}=D\left(\log \frac{\dot{\varepsilon}_{0}}{\dot{\varepsilon}}\right)
$$

where $D$ is a mapping parameter that quantifies the interaction between rate and temperature and maps from a temperature $T$ to a new temperature $T_{0}$ and mapping the strain rate $\dot{\varepsilon}_{0}$ to a new strain rate $\dot{\varepsilon}$ (i.e. a set of data would be comprised of experiments at a reference temperature $T_{0}$ while varying strain rate, and a set of data would consist of experiments at a reference strain rate of $\dot{\varepsilon}_{0}$ with varying temperature).

Essentially, the beta transition seen on the DMA results is shifted up to room temperature by increments of the change in temperature of the beta transition $\left(5^{\circ} \mathrm{C}\right.$ for $\mathrm{PVC}$, or $D$ in equation (2) per decade of strain rate (found from the comparison of test frequencies). By calculating 


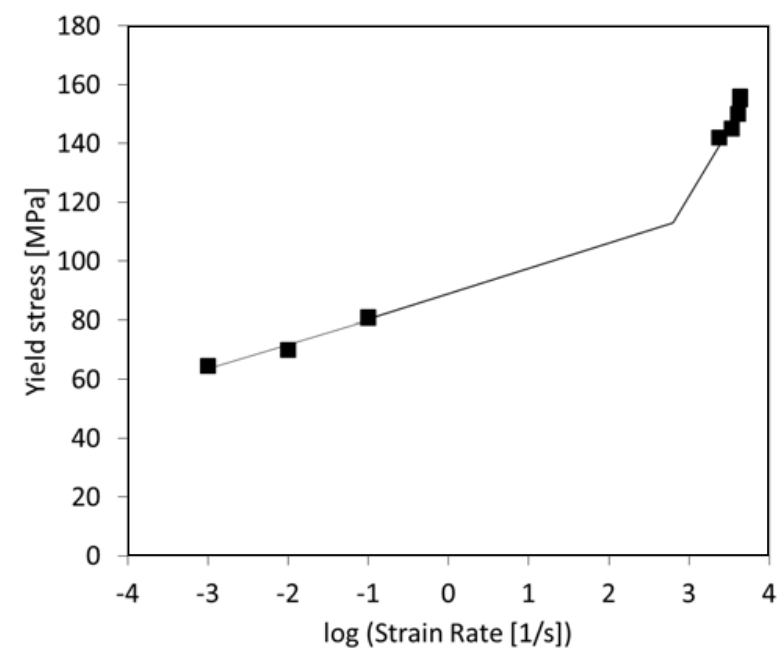

a

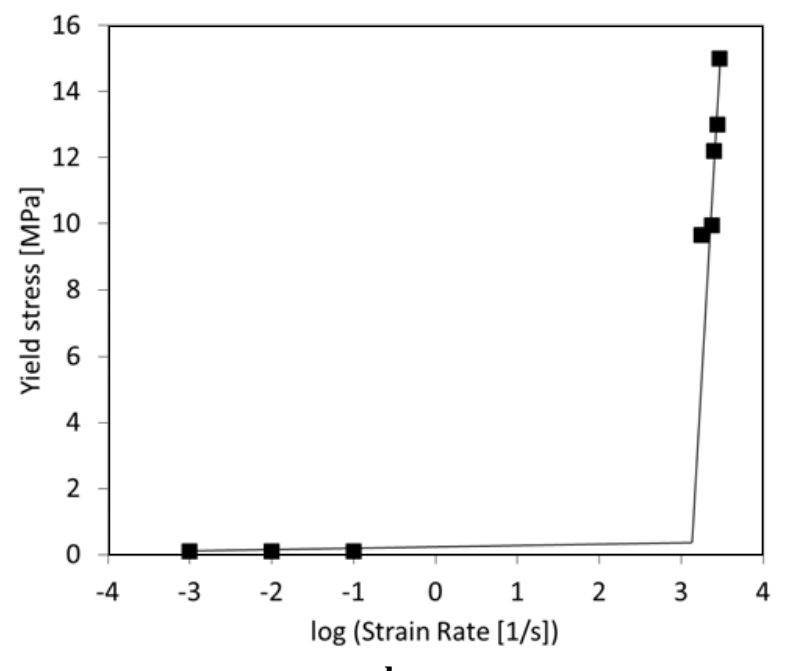

b

Fig. 5. PVC (a) and PPVC (b) yield stress dependence on the logarithm of strain rate.

this shift, the strain rate at which the beta transition is expected to occur at room temperature is found. This was compared with the value of strain rate that would be found by a bilinear fit to the yield stress versus log strain rate curve and calculating where the two lines intersect. The DMA shifting method produced a value of 2.9 for the logarithm of strain rate, or roughly $800 \mathrm{~s}^{-1}$. With reference to figure $5(\mathrm{a})$, we observe that the intersection of the lines fitted to the yield stress data on the same material is 2.8 . There is therefore very close agreement between the values from the two methods.

\section{Conclusions}

Two PVCs with different plasticiser levels have been characterised through uniaxial compression experiments over a broad rage of strain rates and temperatures. DMA characterisation has also been performed on one of these materials. In both materials, the effects of polymer transitions on the rate dependence are clear, the glass transition

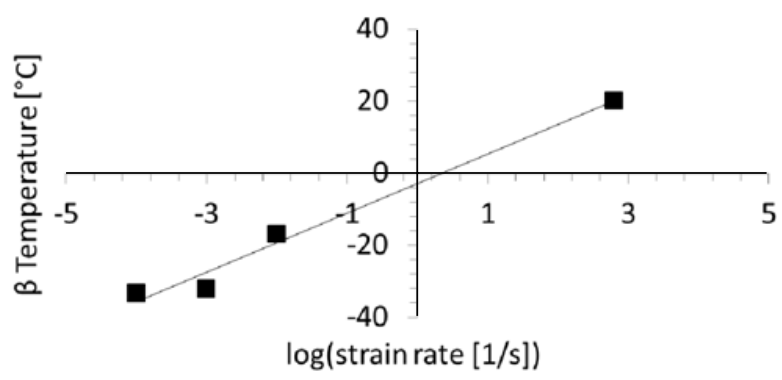

Fig. 6. $\beta$ transition temperature as a function of strain rate. The three data points on the left are from DMA data, the line is fitted to these data and extrapolated to $21^{\circ} \mathrm{C}$ to produce the point on the right.

in the highly plasticised material and the $\beta$ transition in the un-plasticised. Moreover, in the unplasticised PVC, DMA data have been successfully extrapolated to predict the strain rate at which the $\beta$ transition begins to affect the rate dependence of the material.

\section{Acknowledgements}

The authors would like to thank Dr J.L. Jordan and Dr. J.R. Foley for their support of the research, R. Duffin and R. Froud for ongoing technical support, and H. Schiffter and T. Healey for help in conducting DMA testing. M.J. Kendall would also like to acknowledge Dr I. Dyson for help with low rate testing, and Dr D.R. Drodge and Dr E.J. Wielewski for helpful discussions and suggestions. Effort sponsored by the Air Force Office of Scientific Research, Air Force Material Command, USAF, under grant number FA8655-09-1-3088. The US Government is authorized to reproduce and distribute reprints for Governmental purpose notwithstanding any copyright notation thereon. The authors thank Dr R. Pollak for his ongoing support.

\section{References}

1. C.R. Siviour, S.M. Walley, W.G. Proud, J.E. Field, Polymer 46 (2005) 12546-12555.

2. A.D. Mulliken, S.Y. Soong, M.C. Boyce, R.E. Cohen, J. Phys. IV France 134 (2006) 217-223.

3. G.M. Swallowe and S.F. Lee, J. Phys. IV France 110 (2003) 33-38.

4. J.L. Jordan, J.R. Foley, C.R. Siviour, Mech TimeDepend Mater 12 (2008) 249-272.

5. C.R. Siviour, S.M. Walley, W.G. Proud, J.E. Field, J. Phys. IV France 134 (2006) 949-955.

6. C. Bauwens-Crowet, J. Mater. Sci. 8 (1973) 968-979.

7. C.M. Cady, W.R. Blumenthal, G.T. Gray III, D.J. Idar, J.Phys. IV France 110 (2003) 27-32.

8. S. Walley and J. Field, DYMAT J. 1 (1994) 211-227.

9. A.D. Mulliken and M.C. Boyce, Int. J. Solids Struc. 43 (2006) 1331-1356.

10. D.A. Gorham, J. Phys. D: Appl. Phys. 24 (1991) 14891492.

11. A. Trautmann, C.R. Siviour, S.M. Walley, J.E. Field, Int. J. Impact Engng 31 (2005) 523-544. 
12. G.T. Gray III: in ASM Handbook Volume 8, "Mechanical Testing and Evaluation", edited by H. Kuhn and D. Medlin, (ASM International, Materials Park), (2002) 462-476.
13. G.T. Gray III: in ASM Handbook Volume 8, "Mechanical Testing and Evaluation", edited by H. Kuhn and D. Medlin, (ASM International, Materials Park), (2000) 488-496. 